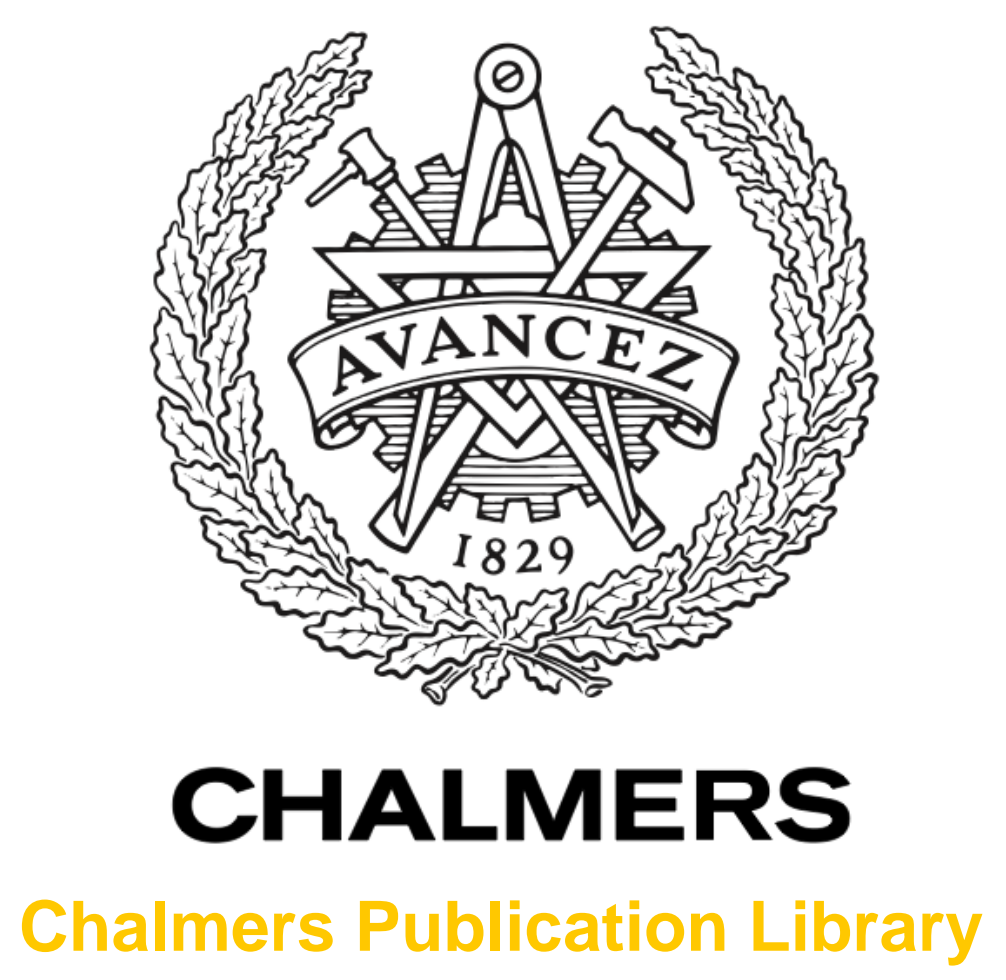

\title{
Analysis of thermal coupling effects in integrated MIMO transmitters
}

This document has been downloaded from Chalmers Publication Library (CPL). It is the author's version of a work that was accepted for publication in:

\section{IEEE MTT-S International Microwave Symposium Digest (ISSN: 0149-645X)}

Citation for the published paper:

Baptista, E. ; Buisman, K. ; Vaz, J. et al. (2017) "Analysis of thermal coupling effects in integrated MIMO transmitters". IEEE MTT-S International Microwave Symposium Digest pp. 75-78.

http://dx.doi.org/10.1109/MWSYM.2017.8058692

Downloaded from: http://publications.lib.chalmers.se/publication/254002

Notice: Changes introduced as a result of publishing processes such as copy-editing and formatting may not be reflected in this document. For a definitive version of this work, please refer to the published source. Please note that access to the published version might require a subscription. 


\title{
Analysis of Thermal Coupling Effects in Integrated MIMO Transmitters
}

\author{
Emanuel Baptista ${ }^{1,2}$, Koen Buisman ${ }^{1}$, João Caldinhas Vaz ${ }^{2,3}$, and Christian Fager ${ }^{1}$ \\ ${ }^{1}$ Chalmers University of Technology, SE-412 96 Göteborg, Sweden \\ ${ }^{2}$ Instituto Superior Técnico and ${ }^{3}$ Instituto de Telecomunicações, P-1049-001 Lisbon, Portugal
}

\begin{abstract}
This paper presents a detailed analysis of thermal coupling and self-heating effects in highly integrated wireless transmitters. A MIMO transmitter prototype consisting of two closely integrated power amplifiers was built and modelled through microwave and thermal characterizations. The thermal behavior was extracted using FEM software and modelled with an equivalent RC network. The PA model was obtained experimentally using a pulsed setup. An RF-thermal simulator was developed and used with the models to predict joint thermal and electrical behavior. Measurements with modulated communication signals were done and compared with the simulator to demonstrate its feasibility for analysis of thermal effects in highly integrated transmitter applications.

Index Terms - power amplifier, thermal effects, MIMO, 5G mobile communication, wireless communication
\end{abstract}

\section{INTRODUCTION}

Active antenna arrays are a key technology to increase capacity and energy efficiency in next generation wireless systems [1]. In such arrays, the separation between antenna elements is dictated by the wavelength, typically $\lambda / 2$ to minimize undesired side-lobes. A very high level of integration is needed to fit all necessary RF and digital electronics into the designated array grid, in particular at mm-wave frequencies which now are being introduced [2]. The temperature increase and interactions caused by heat dissipation from large numbers of closely placed transmitter power amplifiers (PAs) is therefore an integral part of practical active antenna and transmitter circuit design.

Besombes et al. described how a temperature dependent PHD-model can be used to jointly analyze how heat, mismatch, and nonlinear distortion is affecting the operation of a PA under pulsed RF conditions in a radar scenario [3]. Fager et al. extended this analysis to wideband signals and active antenna array applications [4]. Far-field radiation patterns and user signal distortion was presented for a massive MIMO scenario but no experimental validation was given. Ozalas et al. analyzed the electro-thermal coupling in the design of an integrated HBT power amplifier [5]. The thermal coupling impacts on gain compression and low frequency memory effects were presented and analyzed using a commercial simulator, but no experimental validation was presented.

In this work, we build on the results from [3-5] to analyze the impact of self- and mutual heating in highly integrated transmitter circuits. RF and thermal models are developed and integrated into a joint simulation framework. Experimental results, using a dual-path PA are used to validate the simulation results, and to prove the usefulness of the method as a tool for joint thermal/RF co-design in future MIMO transmitters.

\section{ANALYSIS FRAMEWORK}

The analysis presented in this paper is based on the complex envelope simulation framework presented in $[3,4]$. While it can account for PA mismatch and antenna mutual coupling effects, we here focus on thermal interaction between two closely spaced PAs. Fig. 1 presents the analysis framework used:

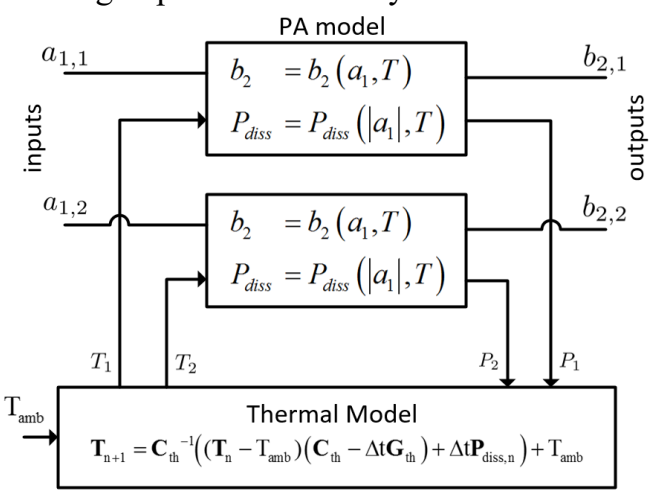

Fig. 1. Electro-thermal two-path MIMO transmitter model used in this work.

In Fig. $1, a_{1}$ and $b_{2}$ represent the $50 \Omega$ normalized PA incident and evanescent voltage waves, respectively. Determination of the model outputs (e.g. $b_{2}$ and $T$ ) requires the joint solution of nonlinear and temperature dependent PA models with a linear thermal model that describes the coupling between the PAs and their environment [4].

\section{A. Power amplifier model}

Focusing here on thermal effects, the output mismatch and RF memory effects are neglected and a simple quasi-static complex envelope polynomial model is used to describe the PA inputand output relationship:

$$
b_{2}\left(a_{1}, T\right)=\sum_{p_{1}=1}^{P_{1}} \alpha_{p_{1}}(T) a_{1}\left|a_{1}\right|^{2\left(p_{1}-1\right)}
$$

where $T$ is the PA temperature and $P_{1}$ the maximum polynomial order. The model temperature dependence is implemented through the complex coefficients $\alpha_{p 1}$. Following [4], the temperature and input signal dependence of the PA power dissipation, $P_{\text {diss }}$, is modeled by a polynomial expression:

$$
P_{\text {diss }}\left(\left|a_{1}\right|, T\right)=P_{d c}+P_{\text {in }}-P_{\text {out }}=\sum_{p_{d}=0}^{P_{d}} \xi_{p d}(T)\left|a_{1}\right|^{p_{d}}
$$

where the dissipated power $P_{\text {in }}$ and $P_{\text {out }}$ represent the power in the $a_{1}$ and $b_{2}$ waves, respectively. $P_{d c}$ is the supply dc power. Again the temperature dependence is introduced through the polynomial coefficients, $\xi_{p d}$. 


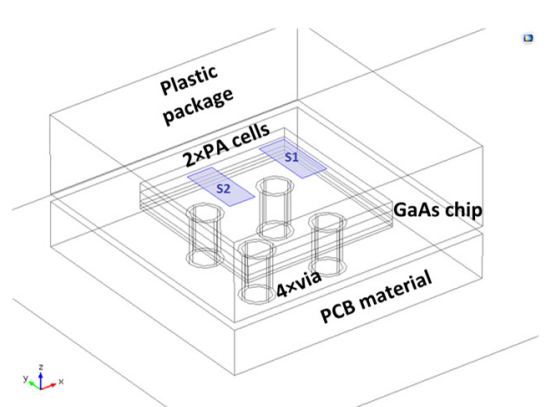

(a) CAD model of packaged dual path PA

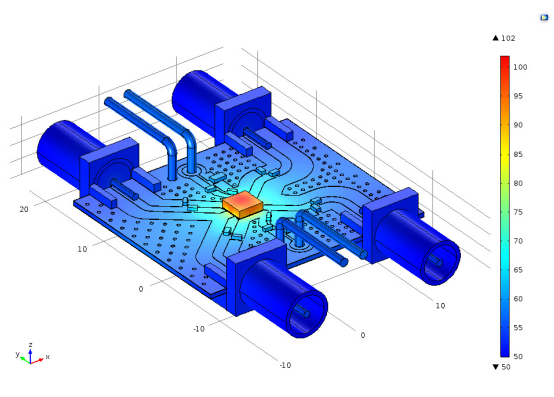

(b) Thermal simulation result for test circuit.

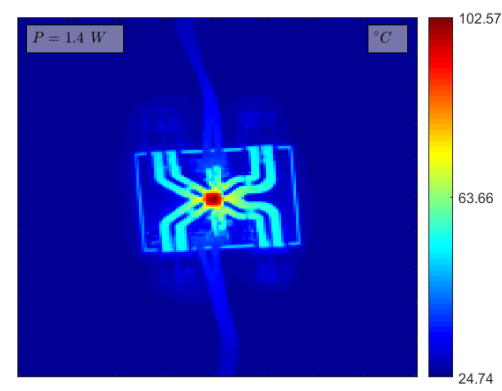

(c) IR camera image.

Fig. 2. Simulation model used for modeling of thermal interactions in the manufactured dual path power amplifier prototype.

\section{B. Thermal model}

A linear heating model is used to relate a constant dissipated power and the resulting temperature:

$$
\mathbf{T}(t)=T_{a m b}+\mathbf{Z}_{t h}(t) \mathbf{P}_{\text {diss }}
$$

where $T_{a m b}$ is the ambient temperature and $\mathbf{Z}_{t h}$ is the thermal impedance matrix, which can be decomposed into an equivalent network of thermal conductances, $\mathbf{G}_{\mathrm{th}}$, and capacitances, $\mathbf{C}_{\mathrm{th}}$. Eq. (3) is later used to find the thermal impedance matrix of the PAs. The temperature in each PA is obtained using a timestepped forward Euler solution:

$$
\mathbf{T}_{\mathrm{n}+1}=\mathbf{C}_{\mathrm{th}}^{-1}\left(\left(\mathbf{T}_{\mathrm{n}}-\mathrm{T}_{\mathrm{amb}}\right)\left(\mathbf{C}_{\mathrm{th}}-\Delta \mathrm{t} \mathbf{G}_{\mathrm{th}}\right)+\Delta \mathrm{t} \mathbf{P}_{\text {diss }, \mathrm{n}}\right)+\mathrm{T}_{\mathrm{amb}}
$$

where $\Delta t$ is the envelope simulation time step. Equation (4) is derived from a nodal analysis using KCL to the network presented in Fig. 6 and by applying a finite difference quotient to the obtained partial differential equations in matrix form. The result obtained can be applied to any RC network. In (4), the power dissipation establishes a direct connection between the PA input signals and the temperature.

\section{MODEL EXTRACTION}

An electro-thermal model of an integrated dual-channel power amplifier prototype will now be presented to illustrate the use of the analysis above.

\section{A. Power amplifier model extraction}

A HMC461 packaged dual-channel 1.7-2.2 GHz PA from Analog Devices is used for the experimental investigations. Inside the package, the HMC461 consists of two closely integrated HBT PA chips, see Fig. 2a. A printed circuit board was realized to enable individual $\mathrm{DC}$ and $\mathrm{RF}$ connections to the two PAs, see Fig. 2b. The electrical grounding and cooling was provided by four via-holes under the package, see Fig. 2a.

The PA was characterized at $2 \mathrm{GHz}$ versus input power and temperature using the setup in Fig. 3. Both the RF and DC bias were pulsed $(200 \mu$ s pulse, $1 \mathrm{~s}$ period) to minimize the influence of device self-heating.

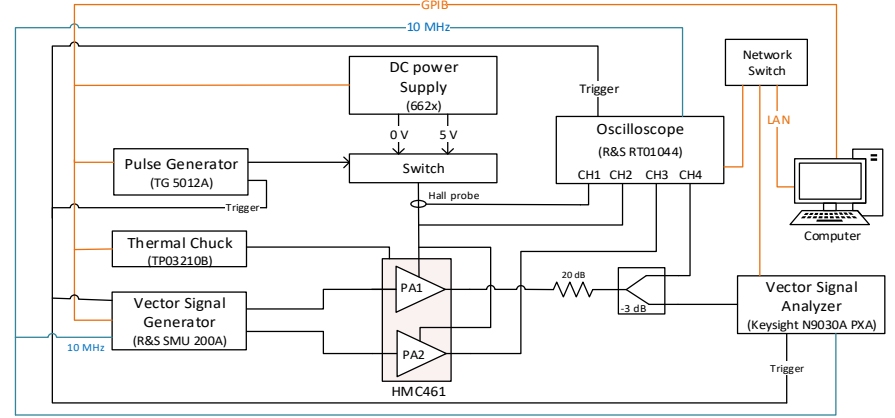

Fig. 3. Setup used for PA modeling and thermal coupling experiments.

A four channel $4 \mathrm{GHz}$ oscilloscope was used to simultaneously capture the PA input and output RF signals, DC voltage and current. The PA models in (1) and (2) were extracted by leastsquares fitting to the experimental RF and DC data, see Fig. 4.
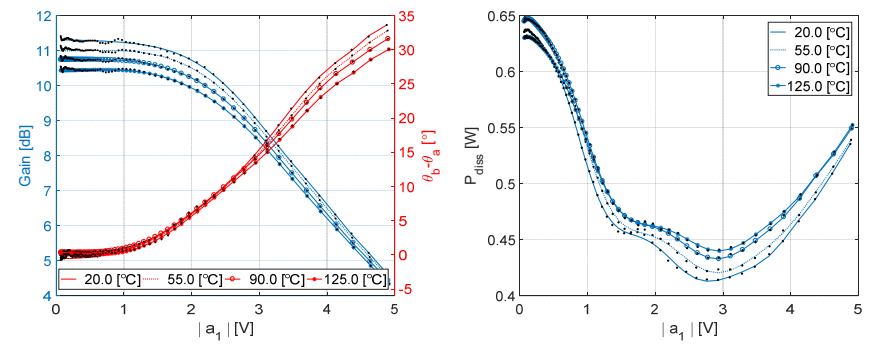

Fig. 4. Measured ( $)$ and modeled (-) AM/AM/PM (left) and dissipated power (right) versus input power and temperature.

\section{B. Thermal model extraction}

A thermal model was developed based on simulations using COMSOL Multiphysics. Thicknesses and thermal proprieties were estimated and assigned to the different layers and components used. Thermally, the PAs are represented by two boundary heat sources $\left(S_{1}\right.$ and $\left.S_{2}\right)$ inside the package, see Fig. 2a. Natural convection was defined around the PCB with a heat transfer to air of $12.7\left[\mathrm{~W} / \mathrm{m}^{2} \cdot \mathrm{K}\right]$, where the value was adjusted to fit steady state IR measurements.

Fig. $2 \mathrm{~b}$ and Fig. $2 \mathrm{c}$ show simulated and measured (IR camera) surface temperature for the manufactured PCB when both PAs dissipate $0.7 \mathrm{~W}$ each. Note that the IR image does not show the correct temperature in metallic regions where the emissivity is low. 

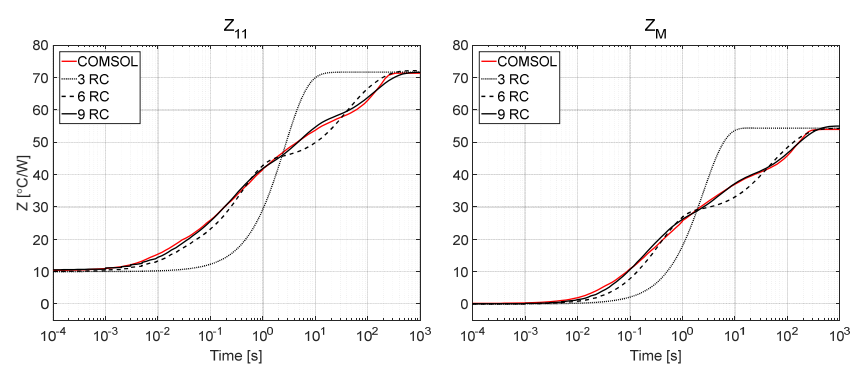

Fig. 5. Simulation and modeling of thermal self- and mutual impedances. $Z_{22}$ is similar to $Z_{11}$ and omitted.

Unit thermal step response simulations have then been used with (2) to determine the thermal coupling between the two PAs in the package. The resulting self- and mutual thermal impedances $\left(Z_{11}, Z_{\mathrm{M}}\right)$ are shown in Fig. 5. This behavior has been approximated by an equivalent temperature independent electrical network model with 9 RC time constants, see Fig. 6. Here, the current sources $\left(\mathrm{P}_{1}\right.$ and $\left.\mathrm{P}_{2}\right)$ represent the dissipated power for each PA in the package while the nodes T1 and T2 represent their temperature.

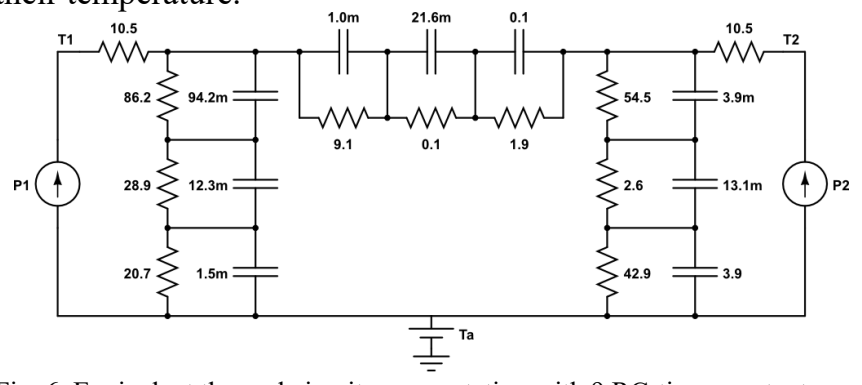

Fig. 6. Equivalent thermal circuit representation with 9 RC-time constants.

\section{RESULTS}

The thermal- and PA models developed in Section III are used with the simulation framework in Section II to analyze the influence of self-heating and thermal coupling in the integrated dual-channel transmitter test circuit. In the first test case, PA1 is turned on at $t=0$ while PA2 is switched off $\left(P_{\text {diss }}=0\right)$. In the other case, both PA1 and PA2 are switched on at $t=0$. Modulated 64-QAM signals with -10 and $-25 \mathrm{dBm}$ input power were applied to PA1 and PA2 inputs, respectively. The experiments were performed at room temperature using the setup in Fig. 3 with the thermal chuck disabled.

\section{A. Temperature}

First, the PA temperature is analyzed versus time for both test cases, see Fig. 7. Clearly, the PA in these cases has several thermal time constants, ranging from the millisecond to $10 \mathrm{~s}$ of second range. Temperature reaches steady state after $600 \mathrm{~s}$. From Fig. 7, we can clearly see the influence of thermal coupling. After 10 s PA1 is $24^{\circ} \mathrm{C}$ hotter when PA2 is active compared to the case when PA2 is off. Likewise, even when not activated, the temperature of PA2 reaches $50^{\circ} \mathrm{C}$. Experimental verification of these results is difficult with the PAs placed inside the package, although measurements by integrated temperature sensors have been evaluated in [6].
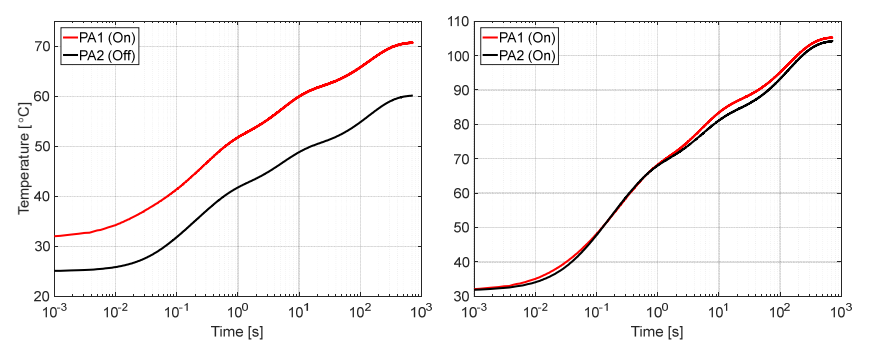

Fig. 7. Investigation of PA temperature vs. time with PA2 switched off (left) and on (right).

\section{B. Gain and error vector magnitude}

While the heating effects are difficult to measure directly, its consequences on the PA characteristics can be accurately characterized. Fig. 8 shows the measured and predicted gain vs. time for PA2 on and off, respectively.

The thermal coupling is clearly reflected in the results. The gain of PA1 decreases by up to $0.5 \mathrm{~dB}$ depending on whether PA2 is on- or off. The self-heating effect is also visible, causing the gain of PA1 to consistently decrease as the device heats up. The heating is also reflected in an increase in RMS error vector magnitude from less $<1 \%$ at startup to $5.6 \%$ and $2.7 \%$ after $100 \mathrm{~s}$ for the two test cases, respectively. This degradation is mainly attributed to the non-constant gain in Fig. 8.

The fact that measured and simulated gain characteristics agree very well proves the validity of the approach for analysis of joint thermal/circuit analysis.

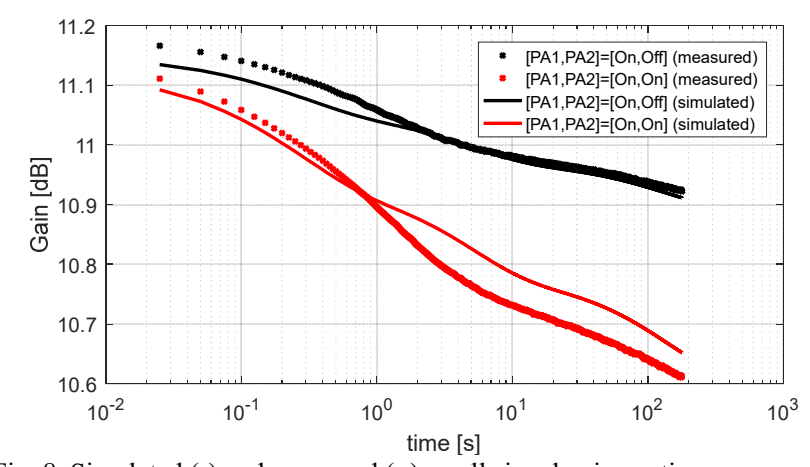

Fig. 8. Simulated (-) and measured $(\times)$ small signal gain vs. time.

\section{CONCLUSIONS}

We have analyzed the impact of temperature and thermal coupling in an integrated dual-path PA prototype. The experimental results show that the self- and mutual heating between neighboring PAs has a significant influence on transmitter gain characteristics, and that it can be well predicted by the proposed electro-thermal modeling procedure. This is of particular importance since both thermal and RF need to be jointly considered when highly integrated active antenna and MIMO transmitters for future wireless systems should be realized.

\section{ACKNOWLEDGEMENT}

The authors thank Dr. Peter Zampardi (Qorvo) for his support. 


\section{REFERENCES}

[1] "5G radio access,". http://www.ericsson.com/res/docs/whitepapers/wp5g.pdf: Ericsson white paper, 2016.

[2] T. S. Rappaport et al., "Millimeter Wave Mobile Communications for 5G Cellular: It Will Work!," IEEE Access, vol. 1, pp. 335-349, 2013.

[3] F. Besombes et al., "Electrothermal behavioral model identification and validation from time domain load pull measurement of a RF power amplifier," Proc. IEEE IMS, pp. 1-3, 2012.
[4] C. Fager et al., "Analysis of thermal effects in active antenna array transmitters using a combined EM/circuit/thermal simulation technique," Proc. 2015 INMMiC, 2015.

[5] M. T. Ozalas, "The Impact of Electro-Thermal Coupling on HBT Power Amplifiers," Proc. 2014 IEEE CSICS, 2014.

[6] D. Gómez et al., "Electro-thermal coupling analysis methodology for RF circuits," Microelectronics Journal, vol. 43, pp. 633-641, 2012. 